\title{
A Rare Case of Adamantinoma in an 11-Year-Old
}

\section{Pouyan Kheirkhah ${ }^{1}$, Bartlomiej Radzik ${ }^{1 *}$, Kyle Sonnabend ${ }^{2}$, Grace Guzman $^{1}$ and Steven Garzon ${ }^{1}$}

${ }^{1}$ Department of Pathology, University of Illinois Hospital and Health System, Chicago, USA

${ }^{2}$ Department of Radiology, University of Illinois Hospital and Health System, Chicago, USA

*Corresponding author: Bartlomiej Radzik, Department of Pathology (MC 847), University of Illinois Hospital and Health System, University of Illinois at Chicago, 840 S. Wood St., Suite 130 CSN, Chicago, IL 60612, USA,

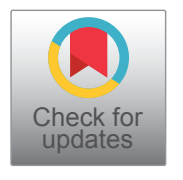
Tel: (312)-996-3886

\begin{abstract}
Adamantinoma is a low-grade malignant lesion most frequently arising in the tibial cortex. Approximately $27 \%$ of adamantinomas present with multifocal lesions in the same bone and $10 \%$ of cases involve the ipsilateral fibula. We describe an 11-year-old male with no significant past medical history who presented to the clinic with a 2-year history of abnormal gait. Two years after the onset of symptoms, a painful mass was noticed on his left shin. Patient biopsy showed a nest of epithelial-like cells arranged in a palisading pattern that stained positive for MCK and Cytokeratin AE1/AE3 and negative for CAM5.2. Radiography demonstrated an expansile, heterogenous lesion arising from the anterior tibial cortex with lucent and sclerotic features. A final diagnosis of adamantinoma was made followed by a complete resection of tumor. Adamantinoma is characterized by intermingled epithelial and osteofibrous components; however, IHC stains are often required to differentiate between osteofibrous dysplasia (OFD), osteofibrous dysplasia-like adamantinoma (OFD-A), and classical adamantinoma. This case highlights the importance of proper bone core biopsy sampling and the characteristic features of this rare pediatric bone tumor.
\end{abstract}

\section{Introduction}

Osteofibrous dysplasia-like adamantinoma is a low-grade malignancy of bone that mostly occurs in patients under 20-years-old and has an incidence of $<1 \%$ [1]. Approximately $27 \%$ of adamantinomas present with multifocal lesions in the same bone and $10 \%$ of cases involve the ipsilateral fibula [2]. Due to similarities in age, radiological appearance, and histological similarities, this entity has recently been described as an intermediate tumor on the spectrum between osteofibrous dysplasia and adamantinoma
[3]. Osteofibrous dysplasia is a benign bone lesion that occurs almost exclusively in pediatric long bone. Histologically it is characterized by irregular bony trabeculae set within bland fibrous stroma organized in a storiform pattern and, by definition, lacks an epithelial component. Adamantinoma is classified into two forms that correlate to prognosis of the tumor. OFD-A is characterized by scattered epithelial elements that and with an average age of incidence of 14 years of age. This tumor, if left untreated can develop into the classic form of adamantinoma which is characterized by bland epithelial cells in clusters with peripheral palisading in fibrous stroma and haphazard osteoid deposition. The sarcomatoid transformation has the worst prognosis and is characterized by highly pleomorphic cells, a high mitotic count, deposition of osteoid and chondroid matrix and no epithelial features. Treatment of osteofibrous dysplasia is either observation or local resection whereas treatment of adamantinoma requires excision with wide resection margins to minimize the risk of recurrence.

\section{Case Presentation}

An 11-year-old male with a past medical history of pes planus, metabolic syndrome and left lower extremity pain and swelling presented to our institution with a 2-year history of abnormal gait. The patient and his mother reported a bump in the anterior leg that had been present for more than a year but became tender to palpation at 6 months prior to presenting to clinic. Physical exam noted a $2 \mathrm{~cm}$ growth on the left midanterior tibia with mild internal rotation of the left lower 


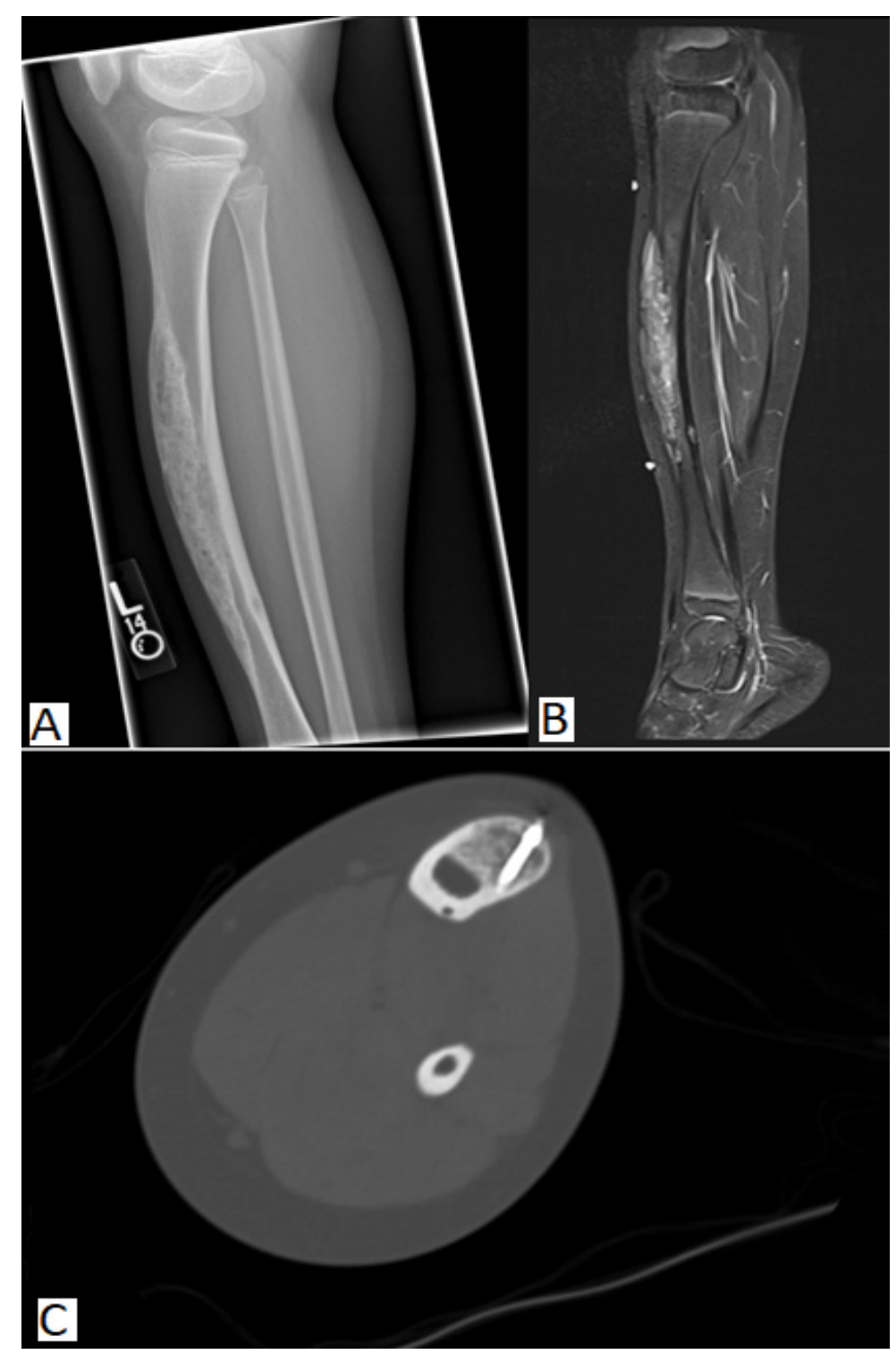

Figure 1: (A) Expansile, heterogenous lesion arising from the anterior tibial cortex with lucent and sclerotic features. Posterior margins are partially obscured by sclerosis, but without periosteal reaction or soft tissue mass; (B) CT showing an infiltrative, $16.6 \mathrm{~cm}$ expansile lesion with its epicenter in the mid-tibial diaphysis with associated tibial bowing anteriorly and poorly defined proximal and distal margins; (C) STIR (short tau inversion recovery) revealing a lesion with heterogenous signal intensity with overall increased STIR signal.

extremity. Plain radiographs (Figure $1 \mathrm{~A}$ ) found focal moderate cortical expansion in the anterior aspect of the mid tibia with cortical irregularity and sclerosis with superimposed numerous small foci of bony lucency. This finding was confirmed with $\mathrm{CT}$ and MRI (Figure $1 \mathrm{~B}$ and Figure $1 \mathrm{C}$ ) to reveal an infiltrative, $16.6 \mathrm{~cm}$ expansile lesion with its epicenter in the mid-tibial diaphysis and was associated tibial bowing anteriorly and poorly defined proximal and distal margins. The mass was described as a mixed lytic and blastic tibial diaphyseal lesion. CT-Guided bone biopsy showed a fibroosseous lesion of thickened woven bone and lamellar bony trabeculae embedded in loose fibrous stroma composed of bland spindle cells. Within the spindle cell stroma scattered clusters and cords of epithelioid cells resembling vessels and epithelial clusters were identified. (Figure 2A and Figure 2B) Immunostains for AE1/AE3 (Figure 3B) showed rare foci of positivity in the form of epithelial clusters; cytokeratin CAM 5.2 was negative (Figure 3A). Immunostains also showed scattered nests and chords of epithelial cells highlighted by 34BE12 and cytokeratin OSCAR. The tumor was treated by excision with wide margins. The patient was managed with allograft placement and reconstruction. A computed tomography scan of the chest, abdomen, and pelvis showed no signs of metastasis.

\section{Discussion}

Adamantinoma is a rare lesion that often presents as a palpable mass in the middle of the tibia or the fibula, but can also occur rarely occur in the mandible, forearm, hands, or feet. This slow growing, low-grade, biphasic tumor accounts for less than $1 \%$ of all bone cancers. The prevalence is more common in young adult males but is extremely rare in childhood [4]. OFD is a benign lesion composed of fibro-osseous tissue. 


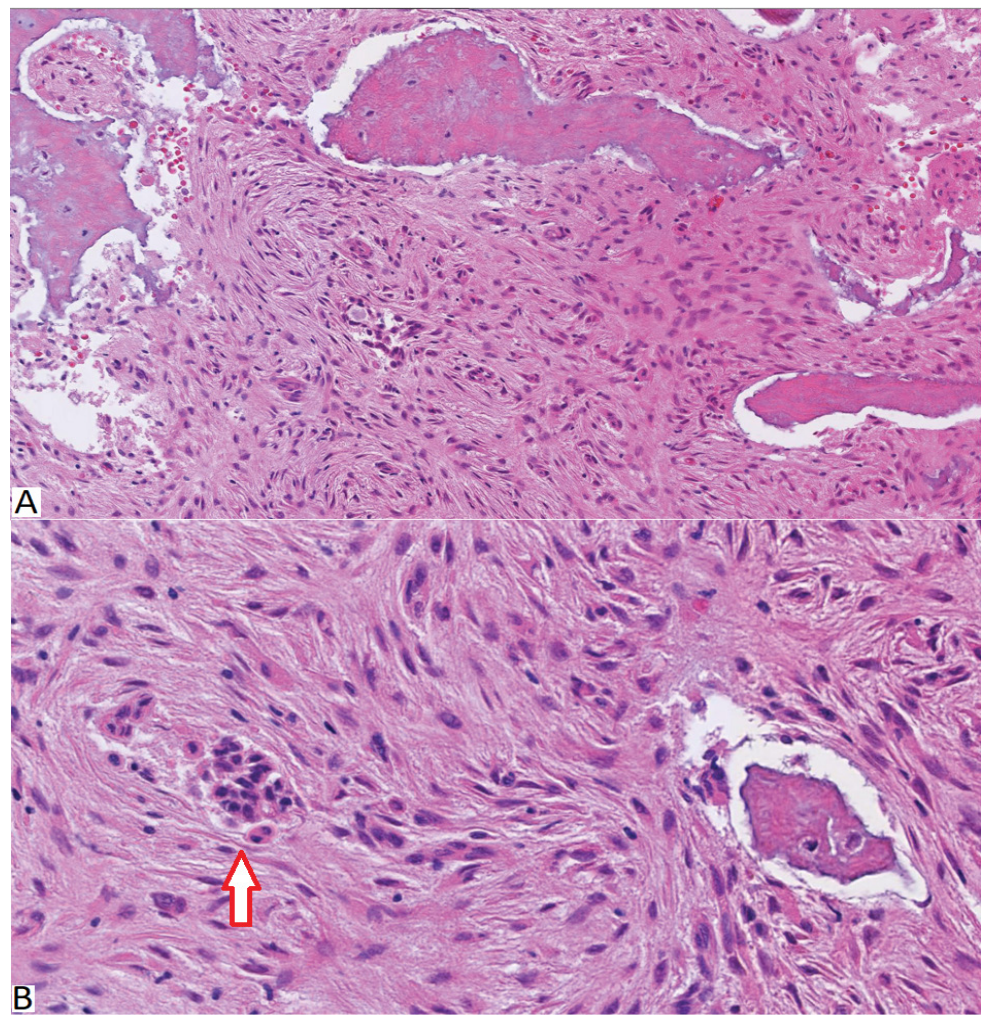

Figure 2: (A) H\&E showing infiltrative fibro-osseous lesion of thickened woven bone and lamellar bony trabeculae embedded in loose fibrous stroma composed of bland spindle cells with occasional clusters of epithelioid cells. Here is a higher magnification showing the bland fibrous appearing spindle cells. The arrow points to epithelioid clusters of cells; (B) High power magnification showing the bland fibrous appearing spindle cells with epithelioid clusters of cells (Arrow).
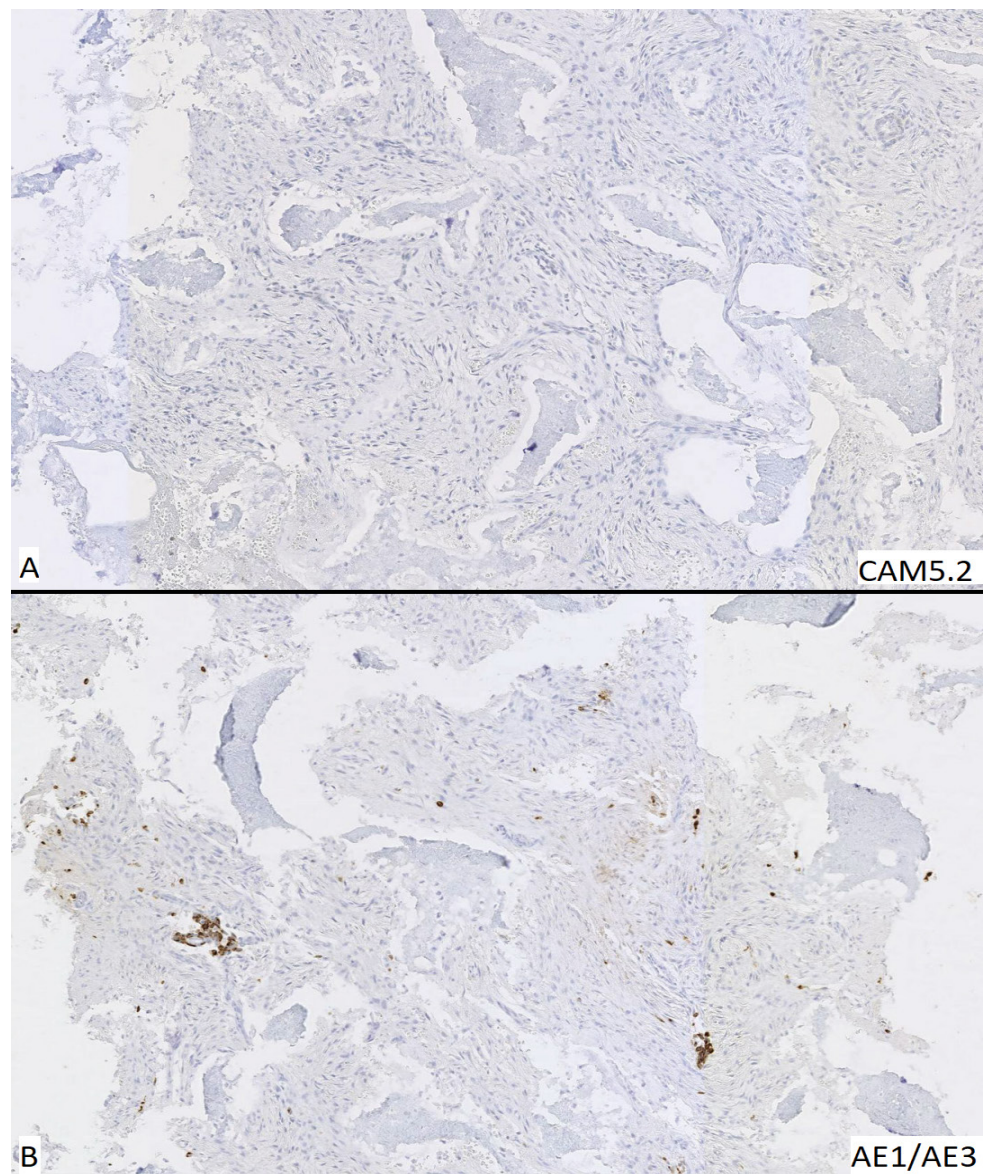

Figure 3: Immunohistochemical stains corresponding to H\&E (A) Cytokeratin CAM5.2 shows no staining of epithelioid cell clusters; (B) Cytokeratin AE1/AE3 shows positive staining for epithelioid cell clusters, confirming the diagnosis of osteofibrous dysplasia-like adamantinoma. 
Adamantinoma is defined as a biphasic tumor with epithelial and fibro-osseous components with local invasion and rare metastasis [4]. The two forms of adamantinoma are OFD-A and classic adamantinoma. OFD-A has a relatively benign course with mixed features of OFD and adamantinoma [5]. OFD-A has a good prognosis and responds well to treatment. It can affect children and adolescents and it lacks the nests and masses of epithelial cells characteristic of the classic form. OFD-A carries a small risk of progression to the classic adamantinoma [6]. Radiographically, OFD and adamantinoma share similar features; however, classic invasive adamantinoma has extensive medullary involvement best seen in MRI. It usually involves most of the medullary bone as compared to minimal involvement by the other two entities [7].

Histologic interpretation plays a major role in differentiation and diagnosis of these entities. Classic adamantinoma should be ruled out in any suspicious fibro-osseous lesion on radiography. An open biopsy or less invasive procedures like core needle biopsy and fine needle biopsy complimented with radiographic confirmation is highly recommended in these cases [8]. However, improper tissue sampling can often result in an incorrect diagnosis of OFD, so it is essential to obtain a sample from the lytic center of the lesion. Based on H\&E staining alone, it may be challenging to differentiate OFD from OFD-A. OFD tends to show a diffuse cytokeratin immunostaining. In contrast, OFD-A shows focal staining of small nests of epithelial cells. Classical adamantinoma, in comparison, shows a biphasic epithelial and osteofibrous pattern on H\&E and is easier to differentiate without immunohistochemistry stains, but cytokeratin immunostaining will reveal large nests of epithelial cells.

Most, et al. hypothesized that these 3 lesions might represent a spectrum of the same disease and are in fact, different stages of a same disease progression [3]. They defied a spectrum of disease with OFD as the benign form, OFD-A as the intermediate form, and classic adamantinoma as the malignant lesion. There has been debate about whether OFD or OFD-A will eventually progress to the classic, invasive form of adamantinoma [8-12]. In contrast to the usual progression hypothesis, two studies proposed that OFD-A is in fact a regressed from classic adamantinoma with some leftover features of the original tumor [13,14]. Management of the lesions widely depends on the histologic diagnosis of the entity. Physicians tend to closely monitor the lesions with no or low malignant potential. Most of the OFD and lesions will go through regression when the patient hits puberty [15]. OFD-A is most often surgically managed either by limb salvage whereas more aggressive surgery with wide resection margins or amputation will be performed for large, deforming, invasive classical adamantinoma $[3,16]$.

\section{References}

1. Ratra A, Wooldridge A, Brindley G (2015) Osteofibrous dysplasia-like adamantinoma of the tibia in a 15-year-old girl. Am J Orthop (Belle Mead NJ) 44: E411-E413.

2. Bloem JL, Taminiau AHM, Eulderink F, Hermans J, Pauwels EK (1988) Radiologic staging of primary bone sarcoma: MR imaging, scintigraphy, angiography and CT correlated with pathologic examination. Radiology 169: 805-810.

3. Most MJ, Sim FH, Inwards CY (2010) Osteofibrous dysplasia and adamantinoma. J Am Acad Orthop Surg 18: 358-366.

4. Van Geel AN, Hazelbag HM, Slingerland R, Vermeulen MI (1997) Disseminating adamantinoma of the tibia. Sarcoma 1: 109-111.

5. Limaiem F, Tafti D, Malik A (2020) Adamantinoma.

6. Kuruvilla G, Steiner GC (1998) Osteofibrous dysplasialike adamantinoma of bone: $A$ report of five cases with immunohistochemical and ultrastructural studies. Hum Pathol 29: 809-814.

7. Bethapudi S, Ritchie DA, Macduff E, Straiton J (2014) Imaging in osteofibrous dysplasia, osteofibrous dysplasialike adamantinoma, and classic adamantinoma. Clin Radiol 69: 200-208.

8. Hazelbag HM, Taminiau AH, Fleuren GJ, Hogendoorn PC (1994) Adamantinoma of the long bones. A clinicopathological study of thirty-two patients with emphasis on histological subtype, precursor lesion, and biological behavior. J Bone Joint Surg Am 76: 1482-1499.

9. Hatori M, Watanabe M, Hosaka M, Sasano H, Narita M, et al. (2006) A classic adamantinoma arising from osteofibrous dysplasia-like adamantinoma in the lower leg: A case report and review of the literature. Tohoku J Exp Med 209: 53-59.

10. Lee RS, Weitzel S, Eastwood DM, Monsell F, Pringle J, et al. (2006) Osteofibrous dysplasia of the tibia. Is there a need for a radical surgical approach? J Bone Joint Surg $\mathrm{Br}$ 88: 658-664.

11. Park YK, Unni KK, McLeod RA, Pritchard DJ (1993) Osteofibrous dysplasia: Clinicopathologic study of 80 cases. Hum Pathol 24: 1339-1347.

12. Springfield DS, Rosenberg AE, Mankin HJ, Mindell ER (1994) Relationship between osteofibrous dysplasia and adamantinoma. Clin Orthop Relat Res (309): 234-244.

13. Czerniak B, Rojas-Corona RR, Dorfman HD (1989) Morphologic diversity of long bone adamantinoma. The concept of differentiated (regressing) adamantinoma and its relationship to osteofibrous dysplasia. Cancer 64: 2319-2334.

14. Ueda Y, Roessner A, Bosse A, Edel G, Böcker W, et al. (1991) Juvenile intracortical adamantinoma of the tibia with predominant osteofibrous dysplasia-like features. Pathol Res Pract 187: 1039-1043.

15. Campanacci M, Laus M (1981) Osteofibrous dysplasia of the tibia and fibula. J Bone Joint Surg Am 63: 367-375.

16. Gleason BC, Liegl-Atzwanger B, Kozakewich HP, Connolly S, Gebhardt MC, et al. (2008) Osteofibrous dysplasia and adamantinoma in children and adolescents: A clinicopathologic reappraisal. Am J Surg Pathol 32: 363-376.
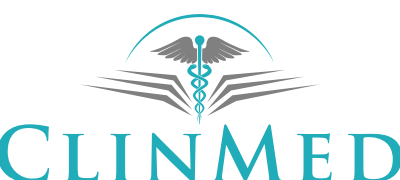

INTERNATIONAL LIBRARY 